PROCEEDINGS OF THE

AMERICAN MATHEMATICAL SOCIETY

Volume 135, Number 4, April 2007, Pages 1007-1015

S 0002-9939(06)08930-1

Article electronically published on November 14, 2006

\title{
A NEW IDENTITY FOR PARSEVAL FRAMES
}

\author{
RADU BALAN, PETER G. CASAZZA, DAN EDIDIN, AND GITTA KUTYNIOK
}

(Communicated by Michael T. Lacey)

\begin{abstract}
In this paper we establish a surprising new identity for Parseval frames in a Hilbert space. Several variations of this result are given, including an extension to general frames. Finally, we discuss the derived results.
\end{abstract}

\section{INTRODUCTION}

Frames are an essential tool for many emerging applications such as data transmission. Their main advantage is the fact that frames can be designed to be redundant while still providing reconstruction formulas. This makes them robust against noise and losses while allowing freedom in design (see, for example, 6, 11). Due to their numerical stability, tight frames and Parseval frames are of increasing interest in applications (see Section 2.1 for definitions). Particularly in image processing, tight frames have emerged as an essential tool (compare [8]). In abstract frame theory, systems constituting tight frames and, in particular, Parseval frames have already been extensively explored [3, 6, 7, 10, 11, 12, yet many questions are still open.

For many years engineers believed that, in applications such as speech recognition, a signal can be reconstructed without information about the phase. In [1] this longstanding conjecture was verified by constructing new classes of Parseval frames for which a signal vector can reconstructed without noisy phase or its estimation. While working on efficient algorithms for signal reconstruction, the authors of [1] discovered a surprising identity for Parseval frames (see 2 for a detailed discussion of the origins of the identity).

Our Parseval Frame Identity can be stated as follows (Theorem 3.2): For any Parseval frame $\left\{f_{i}\right\}_{i \in I}$ in a Hilbert space $\mathbb{H}$, and for every subset $J \subset I$ and every $f \in \mathbb{H}$

$$
\sum_{i \in J}\left|\left\langle f, f_{i}\right\rangle\right|^{2}-\left\|\sum_{i \in J}\left\langle f, f_{i}\right\rangle f_{i}\right\|^{2}=\sum_{i \in J^{c}}\left|\left\langle f, f_{i}\right\rangle\right|^{2}-\left\|\sum_{i \in J^{c}}\left\langle f, f_{i}\right\rangle f_{i}\right\|^{2} .
$$

The proof given here, based on operator theory, admits an elegant extension to arbitrary frames (Theorem 3.1). However, our main focus will be on Parseval frames

Received by the editors June 13, 2005.

2000 Mathematics Subject Classification. Primary 42C15; Secondary 94A12.

Key words and phrases. Bessel sequence, frame, Hilbert space, Parseval frame, Parseval Frame Identity.

The second author was supported by NSF DMS 0405376 .

The third author was supported by NSA MDA 904-03-1-0040.

The fourth author was supported by DFG research fellowship KU 1446/5. 
because of their importance in applications, particularly to signal processing. Several interesting variants of our result are presented; for example, we show that overlapping divisions can also be used. Then the Parseval Frame Identity is discussed in detail; in particular, we derive intriguing equivalent conditions for both sides of the identity to be equal to zero.

\section{Notation AND PRELIminary RESUlts}

2.1. Frames and Bessel sequences. Throughout this paper $\mathbb{H}$ will always denote a Hilbert space and $I$ an indexing set. The finite linear span of a sequence of elements $\left\{f_{i}\right\}_{i \in I}$ of $\mathbb{H}$ will be denoted by $\operatorname{span}\left(\left\{f_{i}\right\}_{i \in I}\right)$. The closure in $\mathbb{H}$ of this set will be denoted by $\overline{\operatorname{span}}\left(\left\{f_{i}\right\}_{i \in I}\right)$.

A system $\left\{f_{i}\right\}_{i \in I}$ in $\mathbb{H}$ is called a frame for $\mathbb{H}$, if there exist $0<A \leq B<\infty$ (lower and upper frame bounds) such that

$$
A\|f\|^{2} \leq \sum_{i \in I}\left|\left\langle f, f_{i}\right\rangle\right|^{2} \leq B\|f\|^{2} \quad \text { for all } f \in \mathbb{H} .
$$

If $A, B$ can be chosen such that $A=B$, then $\left\{f_{i}\right\}_{i \in I}$ is an $A$-tight frame, and if we can take $A=B=1$, it is called a Parseval frame. A Bessel sequence $\left\{f_{i}\right\}_{i \in I}$ is only required to fulfill the upper frame bound estimate but not necessarily the lower estimate. Also, a sequence $\left\{f_{i}\right\}_{i \in I}$ is called a frame sequence, if it is a frame only for $\overline{\operatorname{span}}\left(\left\{f_{i}\right\}_{i \in I}\right)$.

The frame operator $S f=\sum_{i \in I}\left\langle f, f_{i}\right\rangle f_{i}$ associated with $\left\{f_{i}\right\}_{i \in I}$ is a bounded, invertible, and positive mapping of $\mathbb{H}$ onto itself. This provides the frame decomposition

$$
f=S^{-1} S f=\sum_{i \in I}\left\langle f, f_{i}\right\rangle \tilde{f}_{i}=\sum_{i \in I}\left\langle f, \tilde{f}_{i}\right\rangle f_{i}
$$

where $\tilde{f}_{i}=S^{-1} f_{i}$. The family $\left\{\tilde{f}_{i}\right\}_{i \in I}$ is also a frame for $\mathbb{H}$, called the canonical dual frame of $\left\{f_{i}\right\}_{i \in I}$. If $\left\{f_{i}\right\}_{i \in I}$ is a Bessel sequence in $\mathbb{H}$, for every $J \subset I$ we define the operator $S_{J}$ by

$$
S_{J} f=\sum_{i \in J}\left\langle f, f_{i}\right\rangle f_{i}
$$

Finally, we state a known result (see, for example, [9]), since it will be employed several times.

Proposition 2.1. Let $\left\{f_{i}\right\}_{i \in I}$ be a frame for $\mathbb{H}$ with frame operator $S$. For every $f \in \mathbb{H}$, we have

(1) $\left\|\sum_{i \in I}\left\langle f, f_{i}\right\rangle f_{i}\right\|^{2} \leq\|S\| \sum_{i \in I}\left|\left\langle f, f_{i}\right\rangle\right|^{2}$.

(2) $\sum_{i \in I}\left|\left\langle f, f_{i}\right\rangle\right|^{2} \leq\left\|S^{-1}\right\|\left\|\sum_{i \in I}\left\langle f, f_{i}\right\rangle f_{i}\right\|^{2}$.

Moreover, both of these inequalities are best possible.

We note that for Bessel sequences $\left\{f_{i}\right\}_{i \in I}$, the operator $S$ above is well defined, we still call it "the frame operator", and part (1) of Proposition 2.1 remains true. Also, without chance of confusion, we will use $I$ to denote our index set and the identity operator on $\mathbb{H}$.

For more details on frame theory we refer to the survey article [4] and the book 9]. 
2.2. A useful observation. The following observation will be very useful in our proof of the frame identity.

Proposition 2.2. If $S, T$ are operators on $\mathbb{H}$ satisfying $S+T=I$, then $S-T=$ $S^{2}-T^{2}$.

Proof. We compute

$$
S-T=S-(I-S)=2 S-I=S^{2}-\left(I-2 S+S^{2}\right)=S^{2}-(I-S)^{2}=S^{2}-T^{2} .
$$

\section{A NEW IDENTITY}

3.1. General frames. We first study the situation of general frames in $\mathbb{H}$.

Theorem 3.1. Let $\left\{f_{i}\right\}_{i \in I}$ be a frame for $\mathbb{H}$ with canonical dual frame $\left\{\tilde{f}_{i}\right\}_{i \in I}$. Then for all $J \subset I$ and all $f \in \mathbb{H}$ we have

$$
\sum_{i \in J}\left|\left\langle f, f_{i}\right\rangle\right|^{2}-\sum_{i \in I}\left|\left\langle S_{J} f, \tilde{f}_{i}\right\rangle\right|^{2}=\sum_{i \in J^{c}}\left|\left\langle f, f_{i}\right\rangle\right|^{2}-\sum_{i \in I}\left|\left\langle S_{J^{c}} f, \tilde{f}_{i}\right\rangle\right|^{2} .
$$

Proof. Let $S$ denote the frame operator for $\left\{f_{i}\right\}_{i \in I}$. Since $S=S_{J}+S_{J^{c}}$, it follows that $I=S^{-1} S_{J}+S^{-1} S_{J^{c}}$. Applying Proposition 2.2 to the two operators $S^{-1} S_{J}$ and $S^{-1} S_{J^{c}}$ yields

$$
S^{-1} S_{J}-S^{-1} S_{J} S^{-1} S_{J}=S^{-1} S_{J^{c}}-S^{-1} S_{J^{c}} S^{-1} S_{J^{c}} .
$$

Further, for every $f, g \in \mathbb{H}$ we obtain

$$
\left\langle S^{-1} S_{J} f, g\right\rangle-\left\langle S^{-1} S_{J} S^{-1} S_{J} f, g\right\rangle=\left\langle S_{J} f, S^{-1} g\right\rangle-\left\langle S^{-1} S_{J} f, S_{J} S^{-1} g\right\rangle .
$$

Now we choose $g$ to be $g=S f$. Then we can continue the equality (3.2) in the following way:

$$
=\left\langle S_{J} f, f\right\rangle-\left\langle S^{-1} S_{J} f, S_{J} f\right\rangle=\sum_{i \in J}\left|\left\langle f, f_{i}\right\rangle\right|^{2}-\sum_{i \in I}\left|\left\langle S_{J} f, \tilde{f}_{i}\right\rangle\right|^{2} .
$$

Setting equality (3.2) equal to the corresponding equality for $J^{c}$ and using (3.1), we finally get

$$
\sum_{i \in J}\left|\left\langle f, f_{i}\right\rangle\right|^{2}-\sum_{i \in I}\left|\left\langle S_{J} f, \tilde{f}_{i}\right\rangle\right|^{2}=\sum_{i \in J^{c}}\left|\left\langle f, f_{i}\right\rangle\right|^{2}-\sum_{i \in I}\left|\left\langle S_{J^{c}} f, \tilde{f}_{i}\right\rangle\right|^{2} .
$$

3.2. Parseval frames. In the situation of Parseval frames the new identity is of a special form, which moreover enlightens the surprising nature of it.

Theorem 3.2 (Parseval Frame Identity). Let $\left\{f_{i}\right\}_{i \in I}$ be a Parseval frame for $\mathbb{H}$. For every subset $J \subset I$ and every $f \in \mathbb{H}$, we have

$$
\sum_{i \in J}\left|\left\langle f, f_{i}\right\rangle\right|^{2}-\left\|\sum_{i \in J}\left\langle f, f_{i}\right\rangle f_{i}\right\|^{2}=\sum_{i \in J^{c}}\left|\left\langle f, f_{i}\right\rangle\right|^{2}-\left\|\sum_{i \in J^{c}}\left\langle f, f_{i}\right\rangle f_{i}\right\|^{2} .
$$

Proof. We wish to apply Theorem 3.1. Let $\left\{\tilde{f}_{i}\right\}_{i \in I}$ denote the dual frame of $\left\{f_{i}\right\}_{i \in I}$. Since $\left\{f_{i}\right\}_{i \in I}$ is a Parseval frame, its frame operator equals the identity operator 
and hence $\tilde{f}_{i}=f_{i}$ for all $i \in I$. Employing Theorem 3.1 and the fact that $\left\{f_{i}\right\}_{i \in I}$ is a Parseval frame yields

$$
\begin{aligned}
\sum_{i \in J}\left|\left\langle f, f_{i}\right\rangle\right|^{2}-\left\|\sum_{i \in J}\left\langle f, f_{i}\right\rangle f_{i}\right\|^{2} & =\sum_{i \in J}\left|\left\langle f, f_{i}\right\rangle\right|^{2}-\left\|S_{J} f\right\|^{2} \\
& =\sum_{i \in J}\left|\left\langle f, f_{i}\right\rangle\right|^{2}-\sum_{i \in I}\left|\left\langle S_{J} f, f_{i}\right\rangle\right|^{2} \\
& =\sum_{i \in J}\left|\left\langle f, f_{i}\right\rangle\right|^{2}-\sum_{i \in I}\left|\left\langle S_{J} f, \tilde{f}_{i}\right\rangle\right|^{2} \\
& =\sum_{i \in J^{c}}\left|\left\langle f, f_{i}\right\rangle\right|^{2}-\sum_{i \in I}\left|\left\langle S_{J^{c}} f, \tilde{f}_{i}\right\rangle\right|^{2} \\
& =\sum_{i \in J^{c}}\left|\left\langle f, f_{i}\right\rangle\right|^{2}-\left\|S_{J^{c}} f\right\|^{2} \\
& =\sum_{i \in J^{c}}\left|\left\langle f, f_{i}\right\rangle\right|^{2}-\left\|\sum_{i \in J^{c}}\left\langle f, f_{i}\right\rangle f_{i}\right\|^{2} .
\end{aligned}
$$

Note that each side of the Parseval Frame Identity is non-negative, because $S_{J}$ has operator norm at most 1 (see Proposition 2.1 and the remarks that follow it).

A version of the Parseval Frame Identity for overlapping divisions is derived in the following result.

Proposition 3.3. Let $\left\{f_{i}\right\}_{i \in I}$ be a Parseval frame for $\mathbb{H}$. For every $J \subset I$, every $E \subset J^{c}$, and every $f \in \mathbb{H}$, we have

$$
\begin{gathered}
\left\|\sum_{i \in J \cup E}\left\langle f, f_{i}\right\rangle f_{i}\right\|^{2}-\left\|\sum_{i \in J^{c} \backslash E}\left\langle f, f_{i}\right\rangle f_{i}\right\|^{2} \\
=\left\|\sum_{i \in J}\left\langle f, f_{i}\right\rangle f_{i}\right\|^{2}-\left\|\sum_{i \in J^{c}}\left\langle f, f_{i}\right\rangle f_{i}\right\|^{2}+2 \sum_{i \in E}\left|\left\langle f, f_{i}\right\rangle\right|^{2} .
\end{gathered}
$$

Proof. Applying Theorem 3.2 twice yields

$$
\begin{aligned}
& \left\|\sum_{i \in J \cup E}\left\langle f, f_{i}\right\rangle f_{i}\right\|^{2}-\left\|\sum_{i \in J^{c} \backslash E}\left\langle f, f_{i}\right\rangle f_{i}\right\|^{2} \\
& =\sum_{i \in J \cup E}\left|\left\langle f, f_{i}\right\rangle\right|^{2}-\sum_{i \in J^{c} \backslash E}\left|\left\langle f, f_{i}\right\rangle\right|^{2} \\
& =\sum_{i \in J}\left|\left\langle f, f_{i}\right\rangle\right|^{2}-\sum_{i \in J^{c}}\left|\left\langle f, f_{i}\right\rangle\right|^{2}+2 \sum_{i \in E}\left|\left\langle f, f_{i}\right\rangle\right|^{2} \\
& =\left\|\sum_{i \in J}\left\langle f, f_{i}\right\rangle f_{i}\right\|^{2}-\left\|\sum_{i \in J^{c}}\left\langle f, f_{i}\right\rangle f_{i}\right\|^{2}+2 \sum_{i \in E}\left|\left\langle f, f_{i}\right\rangle\right|^{2} .
\end{aligned}
$$

Since each $\lambda$-tight frame can be turned into a Parseval frame by a change of scale, we obtain the following corollary.

Corollary 3.4. Let $\left\{f_{i}\right\}_{i \in I}$ be a $\lambda$-tight frame for $\mathbb{H}$. Then for every $J \subset I$ and every $f \in \mathbb{H}$ we have

$$
\lambda \sum_{i \in J}\left|\left\langle f, f_{i}\right\rangle\right|^{2}-\left\|\sum_{i \in J}\left\langle f, f_{i}\right\rangle f_{i}\right\|^{2}=\lambda \sum_{i \in J^{c}}\left|\left\langle f, f_{i}\right\rangle\right|^{2}-\left\|\sum_{i \in J^{c}}\left\langle f, f_{i}\right\rangle f_{i}\right\|^{2} .
$$

Proof. If $\left\{f_{i}\right\}_{i \in I}$ is a $\lambda$-tight frame for $\mathbb{H}$, then $\left\{\frac{1}{\sqrt{\lambda}} f_{i}\right\}_{i \in I}$ is a Parseval frame for $\mathbb{H}$. Applying Theorem 3.2 proves the result. 
Furthermore, the identity in Theorem 3.2 remains true even for Parseval frame sequences.

Corollary 3.5. Let $\left\{f_{i}\right\}_{i \in I}$ be a Parseval frame sequence in $\mathbb{H}$. Then for every $J \subset I$ and every $f \in \mathbb{H}$ we have

$$
\sum_{i \in J}\left|\left\langle f, f_{i}\right\rangle\right|^{2}-\left\|\sum_{i \in J}\left\langle f, f_{i}\right\rangle f_{i}\right\|^{2}=\sum_{i \in J^{c}}\left|\left\langle f, f_{i}\right\rangle\right|^{2}-\left\|\sum_{i \in J^{c}}\left\langle f, f_{i}\right\rangle f_{i}\right\|^{2} .
$$

Proof. Let $P$ denote the orthogonal projection of $\mathbb{H}$ onto $\overline{\operatorname{span}}\left(\left\{f_{i}\right\}_{i \in I}\right)$. By Theorem 3.2 for every $f \in \mathbb{H}$, we have

$$
\sum_{i \in J}\left|\left\langle P f, f_{i}\right\rangle\right|^{2}-\left\|\sum_{i \in J}\left\langle P f, f_{i}\right\rangle f_{i}\right\|^{2}=\sum_{i \in J^{c}}\left|\left\langle P f, f_{i}\right\rangle\right|^{2}-\left\|\sum_{i \in J^{c}}\left\langle P f, f_{i}\right\rangle f_{i}\right\|^{2} .
$$

Since $\left\langle P f, f_{i}\right\rangle=\left\langle f, P f_{i}\right\rangle=\left\langle f, f_{i}\right\rangle$ for all $i \in I$, the result follows.

We finally remark that Theorem 3.2 is logically equivalent to Theorem 3.1 . Indeed, let $\left\{f_{i}\right\}_{i \in I}$ be a frame for $\mathbb{H}$, with frame operator $S$. It is well known (see, for example, [4, Theorem 4.2]) that $\left\{h_{i}:=S^{-\frac{1}{2}} f_{i}\right\}_{i \in I}$ is a Parseval frame for $\mathbb{H}$. It is easy to check that Theorem 3.2 applied to $\left\{h_{i}\right\}_{i \in I}$ yields Theorem 3.1 .

\section{Discussion of the Parseval Frame Identity}

The identity given in Theorem 3.2 is quite surprising in that the quantities on the two sides of the identity are not comparable to one another in general. For example, if $J$ is the empty set, then the left-hand side of this identity is zero because

$$
\sum_{i \in J}\left|\left\langle f, f_{i}\right\rangle\right|^{2}=0=\left\|\sum_{i \in J}\left\langle f, f_{i}\right\rangle f_{i}\right\|^{2} .
$$

The right-hand side of this identity is also zero, but now because

$$
\sum_{i \in J^{c}}\left|\left\langle f, f_{i}\right\rangle\right|^{2}=\|f\|^{2}=\left\|\sum_{i \in J^{c}}\left\langle f, f_{i}\right\rangle f_{i}\right\|^{2} .
$$

Similarly, if $|J|=1$, then both terms on the left-hand side of this identity may be arbitrarily close to zero, while the two terms on the right-hand side of the identity are nearly equal to $\|f\|^{2}$, and they are canceling precisely enough to produce the identity.

If $\left\{f_{i}\right\}_{i \in I}$ is a Parseval frame for $\mathbb{H}$, then for every $J \subset I$ and every $f \in \mathbb{H}$ we have

$$
\|f\|^{2}=\sum_{i \in J}\left|\left\langle f, f_{i}\right\rangle\right|^{2}+\sum_{i \in J^{c}}\left|\left\langle f, f_{i}\right\rangle\right|^{2}
$$

Hence, one of the two terms on the right-hand side of the above equality is greater than or equal to $\frac{1}{2}\|f\|^{2}$. It follows from Theorem 3.2 that for every $J \subset I$ and every $f \in \mathbb{H}$,

$$
\sum_{i \in J}\left|\left\langle f, f_{i}\right\rangle\right|^{2}+\left\|\sum_{i \in J^{c}}\left\langle f, f_{i}\right\rangle f_{i}\right\|^{2}=\sum_{i \in J^{c}}\left|\left\langle f, f_{i}\right\rangle\right|^{2}+\left\|\sum_{i \in J}\left\langle f, f_{i}\right\rangle f_{i}\right\|^{2} \geq \frac{1}{2}\|f\|^{2} .
$$

We will now see that actually the right-hand side of this inequality is in fact much larger. 
Proposition 4.1. If $\left\{f_{i}\right\}_{i \in I}$ is a Parseval frame for $\mathbb{H}$, then for every $J \subset I$ and every $f \in \mathbb{H}$ we have

$$
\sum_{i \in J}\left|\left\langle f, f_{i}\right\rangle\right|^{2}+\left\|\sum_{i \in J^{c}}\left\langle f, f_{i}\right\rangle f_{i}\right\|^{2} \geq \frac{3}{4}\|f\|^{2} .
$$

Further, we have equality for all $f \in \mathbb{H}$ if and only if $S_{J}=\frac{1}{2} I$.

Proof. By completing the square, we see that

$$
S_{J}^{2}+S_{J^{c}}^{2}=2\left(S_{J}-\frac{1}{2} I\right)^{2}+\frac{1}{2} I
$$

and so

$$
S_{J}^{2}+S_{J^{c}}^{2} \geq \frac{1}{2} I
$$

with equality if and only if $S_{J}=\frac{1}{2} I$.

Since $S_{J}+S_{J^{c}}=I$, it follows that $S_{J}+S_{J^{c}}^{2}+S_{J^{c}}+S_{J}^{2} \geq \frac{3}{2} I$. Applying Proposition 2.2 to $S=S_{J}$ and $T=S_{J^{c}}$ yields $S_{J}+S_{J^{c}}^{2}=S_{J^{c}}+S_{J}^{2}$. Thus

$$
2\left(S_{J}+S_{J^{c}}^{2}\right)=S_{J}+S_{J^{c}}^{2}+S_{J^{c}}+S_{J}^{2} \geq \frac{3}{2} I .
$$

Finally, for every $f \in \mathbb{H}$ we have

$\sum_{i \in J}\left|\left\langle f, f_{i}\right\rangle\right|^{2}+\left\|\sum_{i \in J^{c}}\left\langle f, f_{i}\right\rangle f_{i}\right\|^{2}=\left\langle S_{J} f, f\right\rangle+\left\langle S_{J^{c}} f, S_{J^{c}} f\right\rangle=\left\langle\left(S_{J}+S_{J^{c}}^{2}\right) f, f\right\rangle \geq \frac{3}{4}\|f\|^{2}$.

From above, we clearly have equality for all $f \in \mathbb{H}$ if and only if $S_{J}=\frac{1}{2} I$.

We remark that a key part of the proof of Proposition 4.1 extends in a straightforward manner to prove the following fact. Let $\left\{f_{i}\right\}_{i \in I}$ be a Parseval frame for $\mathbb{H}$. Let $\left\{J_{k}\right\}_{k=1}^{n}$ be a finite partition of the index set $I$ and let $S_{k}:=S_{J_{k}}$ for each $k$. Then

$$
S_{1}^{2}+\cdots+S_{n}^{2} \geq \frac{1}{n} I
$$

with equality if and only if $S_{j}=S_{k}$, for all $j, k \in\{1, \ldots, n\}$; if and only if $S_{j}=\frac{1}{n} I$, for all $j \in\{1, \ldots, n\}$.

Let $\left\{f_{i}\right\}_{i \in I}$ be a $\lambda$-tight frame for $\mathbb{H}$. Reformulating Corollary 3.4 yields that for every $J \subset I$ and every $f \in \mathbb{H}$ we have

$$
\lambda \sum_{i \in J}\left|\left\langle f, f_{i}\right\rangle\right|^{2}-\lambda \sum_{i \in J^{c}}\left|\left\langle f, f_{i}\right\rangle\right|^{2}=\left\|\sum_{i \in J}\left\langle f, f_{i}\right\rangle f_{i}\right\|^{2}-\left\|\sum_{i \in J^{c}}\left\langle f, f_{i}\right\rangle f_{i}\right\|^{2} .
$$

We intend to study when both sides of this equality equal zero, which is closely related to questions concerning extending a frame to a tight frame. The proofs of these results use the next lemma as a main ingredient.

Lemma 4.2. Let $\left\{f_{i}\right\}_{i \in I}$ and $\left\{g_{i}\right\}_{i \in K}$ be Bessel sequences in $\mathbb{H}$ with frame operators $S$ and $T$, respectively. If $S=T$, then

$$
\overline{\operatorname{span}}\left(\left\{f_{i}\right\}_{i \in I}\right)=\overline{\operatorname{span}}\left(\left\{g_{i}\right\}_{i \in K}\right) .
$$

Proof. For any $f \in \mathbb{H}$, we have

$$
\sum_{i \in I}\left|\left\langle f, f_{i}\right\rangle\right|^{2}=\langle S f, f\rangle=\langle T f, f\rangle=\sum_{i \in K}\left|\left\langle f, g_{i}\right\rangle\right|^{2} .
$$

It follows that $f \perp f_{i}$ for all $i \in I$ if and only if $f \perp g_{i}$ for all $i \in K$. 
The following result is an immediate consequence of Lemma 4.2 and the above reformulation of Corollary 3.4 .

Proposition 4.3. Let $\left\{f_{i}\right\}_{i \in I}$ be a $\lambda$-tight frame for $\mathbb{H}$ and $J \subset I$. If $\left\|S_{J} f\right\|=$ $\left\|S_{J^{c}} f\right\|$, for all $f \in \mathbb{H}$, then

$$
\overline{\operatorname{span}}\left(\left\{f_{i}\right\}_{i \in J}\right)=\overline{\operatorname{span}}\left(\left\{f_{i}\right\}_{i \in J^{c}}\right) .
$$

It is well known that given a frame $\left\{f_{i}\right\}_{i \in I}$ for a Hilbert space $\mathbb{H}$, there exists a sequence (and in fact there are many such sequences) $\left\{g_{i}\right\}_{i \in K}$ so that $\left\{f_{i}\right\}_{i \in I} \cup$ $\left\{g_{i}\right\}_{i \in K}$ is a tight frame (see, for example, [5]). We will now see that, if we choose two different families to extend $\left\{f_{i}\right\}_{i \in I}$ to a tight frame, then these new families have several important properties in common.

Proposition 4.4. Let $\left\{f_{i}\right\}_{i \in I}$ be a frame for $\mathbb{H}$. Assume that $\left\{f_{i}\right\}_{i \in I} \cup\left\{g_{i}\right\}_{i \in K}$ and $\left\{f_{i}\right\}_{i \in I} \cup\left\{h_{i}\right\}_{i \in L}$ are both $\lambda$-tight frames. Then the following conditions hold:

(1) For every $f \in \mathbb{H}, \sum_{i \in K}\left|\left\langle f, g_{i}\right\rangle\right|^{2}=\sum_{i \in L}\left|\left\langle f, h_{i}\right\rangle\right|^{2}$.

(2) For every $f \in \mathbb{H}, \sum_{i \in K}\left\langle f, g_{i}\right\rangle g_{i}=\sum_{i \in L}\left\langle f, h_{i}\right\rangle h_{i}$.

(3) $\overline{\operatorname{span}}\left(\left\{g_{i}\right\}_{i \in K}\right)=\overline{\operatorname{span}}\left(\left\{h_{i}\right\}_{i \in L}\right)$.

Proof. For all $f \in \mathbb{H}$, we have

$$
\sum_{i \in I}\left|\left\langle f, f_{i}\right\rangle\right|^{2}+\sum_{i \in K}\left|\left\langle f, g_{i}\right\rangle\right|^{2}=\lambda\|f\|^{2}=\sum_{i \in I}\left|\left\langle f, f_{i}\right\rangle\right|^{2}+\sum_{i \in L}\left|\left\langle f, h_{i}\right\rangle\right|^{2} .
$$

This yields (1).

Similarly, for all $f \in H$,

$$
\sum_{i \in I}\left\langle f, f_{i}\right\rangle f_{i}+\sum_{i \in K}\left\langle f, g_{i}\right\rangle g_{i}=\lambda f=\sum_{i \in I}\left\langle f, f_{i}\right\rangle f_{i}+\sum_{i \in L}\left\langle f, h_{i}\right\rangle h_{i},
$$

which proves (2).

Condition (3) follows immediately from (2) and Lemma 4.2 .

In the next theorem we will derive many equivalent conditions for both sides of the Parseval Frame Identity (Theorem 3.2) to equal zero. For this, we first need a result concerning the operators $S_{J}, S_{J^{c}}$.

Proposition 4.5. Let $\left\{f_{i}\right\}_{i \in I}$ be a Parseval frame for $\mathbb{H}$. For any $J \subset I, S_{J} S_{J^{c}}$ is a positive self-adjoint operator on $\mathbb{H}$ which satisfies

$$
S_{J}-S_{J}^{2}=S_{J} S_{J^{c}} \geq 0 .
$$

Proof. Clearly,

$$
S_{J}-S_{J}^{2}=S_{J}\left(I-S_{J}\right)=S_{J} S_{J^{c}} .
$$

Moreover, since $S_{J}$ and $S_{J^{c}}$ are commuting positive, self-adjoint operators, it follows that $S_{J} S_{J^{c}}$ is also positive and self-adjoint.

Note that for any positive operator $T$ on a Hilbert space $\mathbb{H}$ and any $f \in \mathbb{H}$, $T f=0$ implies $\langle T f, f\rangle=0$. The converse of this is also true. If $\langle T f, f\rangle=0$, then by a simple calculation

$$
\langle T f, f\rangle=\left\langle T^{1 / 2} f, T^{1 / 2} f\right\rangle=\left\|T^{1 / 2} f\right\|^{2}=0 .
$$

So $T^{1 / 2} f=0$, and hence $T f=0$. Noting that one side of the Parseval Frame Identity is zero if and only if the other side is, we are led to the following result. 
Theorem 4.6. Let $\left\{f_{i}\right\}_{i \in I}$ be a Parseval frame for $\mathbb{H}$. For each $J \subset I$ and $f \in \mathbb{H}$, the following conditions are equivalent:

(1) $\sum_{i \in J}\left|\left\langle f, f_{i}\right\rangle\right|^{2}=\left\|\sum_{i \in J}\left\langle f, f_{i}\right\rangle f_{i}\right\|^{2}$.

(2) $\sum_{i \in J^{c}}\left|\left\langle f, f_{i}\right\rangle\right|^{2}=\left\|\sum_{i \in J^{c}}\left\langle f, f_{i}\right\rangle f_{i}\right\|^{2}$.

(3) $\sum_{i \in J}\left\langle f, f_{i}\right\rangle f_{i} \perp \sum_{i \in J^{c}}\left\langle f, f_{i}\right\rangle f_{i}$.

(4) $f \perp S_{J} S_{J^{c}} f$.

(5) $S_{J} f=S_{J}^{2} f$.

(6) $S_{J} S_{J^{c}} f=0$.

Proof. $(1) \Leftrightarrow(2)$ : This is follows immediately from Theorem 3.2 ,

$(3) \Leftrightarrow(4)$ : This is proven by the following equality:

$$
\left\langle\sum_{i \in J}\left\langle f, f_{i}\right\rangle f_{i}, \sum_{i \in J^{c}}\left\langle f, f_{i}\right\rangle f_{i}\right\rangle=\left\langle S_{J} f, S_{J^{c}} f\right\rangle=\left\langle f, S_{J} S_{J^{c}} f\right\rangle .
$$

$(5) \Leftrightarrow(6)$ : This follows from Proposition 4.5.

$(1) \Leftrightarrow(5)$ : We have

$$
\sum_{i \in J}\left|\left\langle f, f_{i}\right\rangle\right|^{2}-\left\|\sum_{i \in J}\left\langle f, f_{i}\right\rangle f_{i}\right\|^{2}=\left\langle S_{J} f, f\right\rangle-\left\langle S_{J} f, S_{J} f\right\rangle=\left\langle\left(S_{J}-S_{J}^{2}\right) f, f\right\rangle .
$$

By Proposition 4.5. $S_{J}-S_{J}^{2} \geq 0$. Therefore the right-hand side of the above equality is zero if and only if $\left(S_{J}-S_{J}^{2}\right) f=0$ by our discussion preceding the proposition.

$(1) \Rightarrow(4)$ : By $(1),\left\langle S_{J} f, f\right\rangle=\left\langle S_{J} f, S_{J} f\right\rangle$. Hence $\left\langle S_{J} S_{J^{c}} f, f\right\rangle=\left\langle\left(S_{J}-S_{J}^{2}\right) f, f\right\rangle=$ 0 , which implies (4).

$(4) \Rightarrow(6)$ : By Proposition 4.5, we have that $S_{J} S_{J^{c}} \geq 0$. Thus $\left\langle S_{J} S_{J^{c}} f, f\right\rangle=0$ if and only if $S_{J} S_{J^{c}} f=0$ by the discussion preceding this proposition.

\section{ACKNOWLEDGMENTS}

An announcement for this paper appeared in 2.

The authors wish to thank Alex Petukhov for interesting discussions concerning this paper. Petukhov also provided us with an alternative matrix proof of the Parseval Frame Identity (Theorem 3.2). We also thank Chris Lennard for useful discussions. Lennard also provided us with an alternative proof of the Parseval Frame Identity obtained by expanding both sides as infinite series and comparing the outcome.

We further thank the anonymous referee for very valuable suggestions which improved the paper.

\section{REFERENCES}

1. R. Balan, P.G. Casazza, D. Edidin, Signal reconstruction without noisy phase, Appl. Comput. Harmon. Anal. 20 (2006), 345-356. MR2224902

2. R. Balan, P.G. Casazza, D. Edidin, and G. Kutyniok, Decompositions of frames and a new frame identity, Wavelets XI (San Diego, CA, 2005), 379-388, SPIE Proc. 5914, SPIE, Bellingham, WA, 2005.

3. J.J. Benedetto and M. Fickus, Finite normalized tight frames, Adv. Comput. Math. 18 (2003), 357-385. MR 1968126 (2004c:42059)

4. P.G. Casazza, The art of frame theory, Taiwanese J. of Math. 4 (2000), 129-201. MR 1757401 (2001f:42046) 
5. P.G. Casazza, Custom building finite frames, in: Wavelets, frames and operator theory, 61-86, Contemp. Math., 345, Amer. Math. Soc., Providence, RI, 2004. MR2066822 (2005f:42078)

6. P.G. Casazza and J. Kovaĉević, Equal-norm tight frames with erasures, Adv. Comput. Math. 18 (2003), 387-430. MR1968127 (2004e:42046)

7. P.G. Casazza and G. Kutyniok, A generalization of Gram-Schmidt orthogonalization generating all Parseval frames, Adv. Comput. Math., to appear.

8. R.H. Chan, S.D. Riemenschneider, L. Shen, Z. Shen, Tight frame: an efficient way for high-resolution image reconstruction, Appl. Comput. Harmon. Anal. 17 (2004), 91-115. MR2067917 (2005h:94006)

9. O. Christensen, An Introduction to Frames and Riesz Bases, Birkhäuser, Boston (2003). MR.1946982 (2003k:42001)

10. Y.C. Eldar and G.D. Forney, Jr., Optimal tight frames and quantum measurement, IEEE Trans. Inform. Theory 48 (2002), 599-610. MR1889971 (2003c:94006)

11. V.K. Goyal, J. Kovačević, and J.A. Kelner, Quantized frame expansions with erasures, Appl. Comput. Harmon. Anal. 10 (2001), 203-233. MR:1829801 (2002h:94012)

12. R. Vale and S. Waldron, Tight frames and their symmetries, Constr. Approx. 21 (2005), 83-112. MR2105392(2005h:42063)

Siemens Corporate Research, 755 College Road East, Princeton, New Jersey 08540

E-mail address: radu.balan@siemens.com

Department of Mathematics, University of Missouri, Columbia, Missouri 65211

E-mail address: pete@math.missouri.edu

Department of Mathematics, University of Missouri, Columbia, Missouri 65211

E-mail address: edidin@math.missouri.edu

Institute of Mathematics, Justus-Liebig-University Giessen, 35392 Giessen, Germany

E-mail address: gitta.kutyniok@math.uni-giessen.de 